\title{
SUCCĖS OU ÉCHEC ? LA MÉMOIRE DIVISÉE DU SABORDAGE DE TOULON
}

\author{
Thomas Vaisset, Philippe Vial
}

Armée de terre | «Inflexions »

$2020 / 3 \mathrm{~N}^{\circ} 45 \mid$ pages 45 à 60

ISSN $1772-3760$

DOI 10.3917/infle.045.0045

Article disponible en ligne à l'adresse :

https://www.cairn.info/revue-inflexions-2020-3-page-45.htm

Distribution électronique Cairn.info pour Armée de terre.

(C) Armée de terre. Tous droits réservés pour tous pays.

La reproduction ou représentation de cet article, notamment par photocopie, n'est autorisée que dans les limites des conditions générales d'utilisation du site ou, le cas échéant, des conditions générales de la licence souscrite par votre établissement. Toute autre reproduction ou représentation, en tout ou partie, sous quelque forme et de quelque manière que ce soit, est interdite sauf accord préalable et écrit de l'éditeur, en dehors des cas prévus par la législation en vigueur en France. Il est précisé que son stockage dans une base de données est également interdit. 


\section{SUCAĖS OU ÉCHEC? LA MÉMOIRE DIVISÉE DU SABORDAGE DE TOULON}

Toulon, 27 novembre 1942 : échappant in extremis à la mainmise allemande, une grande partie des forces navales de Vichy se saborde dans la rade. Devenue symbole d'un échec absolu et déshonorant, cette tragédie constitue aujourd'hui une sorte de pendant naval à la débâcle de 1940. Le sabordage en a le caractère soudain, brutal et apparemment irrémédiable. La flotte qui disparaît est en effet, pour l'essentiel, composée du meilleur de la marine de Vichy, les Forces de haute mer (FHM). À la honte de la défaite s'ajoute l'opprobre de la passivité, car les bâtiments sont coulés sans avoir été vaincus, ni même avoir livré bataille.

Pourtant, l'événement est loin d'être immédiatement perçu de cette manière. C'est d'abord le soulagement qui prévaut chez les Alliés. Une force qui aurait pu renforcer de manière importante le potentiel du Reich a échappé de manière spectaculaire à sa mainmise... G'est une gifle et une humiliation pour les autorités allemandes, dont la tentative de hold-up naval (opération Lila), dans le prolongement de l'invasion de la zone libre (opération Anton), a échoué. En dépit de la satisfaction d'Hitler, qui voulait moins capturer la principale flotte française que la neutraliser, si échec il y a, il est pour le Reich et ses soutiens, en particulier les milieux collaborationnistes.

C'est seulement de manière graduelle que le sabordage du 27 novembre 1942 va se transformer en échec cuisant aux yeux de la majorité des observateurs. Un retournement qui, pour autant, ne peut être complet, car l'ambivalence de l'événement interdit toute lecture univoque de cet «événement monstre», pour reprendre l'expression de Pierre Nora ${ }^{1}$. Dans son principe même, un sabordage réussi est un coup ultime porté à l'adversaire autant que la reconnaissance définitive de sa supériorité et un aveu d'impuissance. Toulon est ainsi, à jamais, rien qu'un échec et plus qu'un échec ${ }^{2}$.

1. P. Nora, "L'événement monstre 》, Communications n 18, 1972, pp. 162-172.

2. Pour reprendre en l'adaptant, le titre de l'étude dont est tiré cet article, présentée ici dans une version remaniée et enrichie : T. Vaisset et Ph.. Vial, «Toulon, 27 novembre 1942 : rien qu'une défaite ou plus qu'une défaite?», in C. Defrance, C. Horel et F.-X. Nérard (dir.), Vaincus! Histoires de défaites. Europe XIX'-XX siècle, Paris, Nouveau Monde Éditions, 2016, pp. 29-51. 


\section{Toulon : le sabordage de la flotte française?}

Le bilan du drame est connu. Il est impressionnant. Plus d'une centaine de bâtiments a été volontairement détruite, soit près de deux cent trente-cinq mille tonnes. À l'exception de cinq sous-marins et d'un baliseur qui, ignorant délibérément les ordres, ont réussi à prendre le large, $90 \%$ des unités au mouillage disparaissent sans combat. En quelques heures, la marine enregistre $60 \%$ des pertes qu'elle subira pendant toute la durée du conflit ${ }^{3}$.

Pourtant, contrairement à une idée reçue, aussi fausse que tenace, ce sabordage n'est pas celui de l'ensemble de la flotte française. Les destructions correspondent à environ $40 \%$ de son tonnage lors de l'entrée en guerre. Le sabordage n'est pas non plus celui de la marine, une expression qui n'a plus guère de sens fin novembre 1942. À cette époque, le pavillon tricolore flotte en effet sur des bâtiments qui relèvent de trois autorités différentes.

Avec trente mille tonnes, les Forces navales françaises libres (FNFL) représentent la minorité qui n'a jamais cessé le combat. De son côté, la marine de Vichy est un assemblage composite. Après le sabordage, elle est formée des Forces navales d'Extrême-Orient (treize mille tonnes), de l'escadre des Antilles (trente-huit tonnes) et de la Force X stationnée à Alexandrie (soixante-cinq mille tonnes), soit cent seize mille tonnes environ. Mais les premières sont isolées en Indochine, tandis que les deux autres sont neutralisées par les Américains ou les Britanniques. Enfin, les récents événements d'Afrique du Nord ont donné naissance à un troisième ensemble, composé des bâtiments ayant survécu aux débarquements anglo-américains et renforcé, à la veille du sabordage, par le ralliement de la flotte d'Afrique occidentale française (AOF). Ces cent trente-cinq mille tonnes dépendent des autorités d'Alger, qui viennent de rompre avec Vichy sans pour autant rallier Londres : on va bientôt désigner ces marins comme les «Barbaresques».

Le sabordage entraîne ainsi la destruction de moins de la moitié du tonnage français alors en service. Par ailleurs, ce qui disparaît à Toulon est loin d'être d'égale valeur. Les Forces de haute mer - trente-huit bâtiments seulement, mais plus de la moitié du tonnage - voisinent avec un groupe hétérogène de cent trente-cinq unités, parmi lesquelles une cinquantaine de bâtiments de servitude ou de soutien. Dans ce groupe, les navires de combat sont, pour la plupart, en «gardiennage d'armistice» ou en réparation, c'est-à-dire sans valeur opérationnelle.

3. Service historique de la Défense, archives de la marine conservées à Vincennes (désormais SHD-MV), 3BB² SEC 114, note non numérotée et non signée "Historique du tonnage de la marine militaire de 1939 à 1946 ". 
Néanmoins, qualitativement, c'est bien la majorité des meilleures unités qui disparaît. L'intégralité des FHM, le fer de lance de la marine de Vichy, s'est en effet sabordée. Outre le navire de ligne Strasbourg, les pertes concernent essentiellement les croiseurs et les contre-torpilleurs, qui comptaient parmi les grandes réussites des programmes navals de l'entre-deux-guerres. Mais les bâtiments coulés à Toulon ne sont plus de nature à bouleverser l'équilibre des forces en Europe. Atout capital, avec l'empire, en juin 1940, la flotte ne représente plus qu'une carte secondaire à l'automne 1942 car, entretemps, les conditions de la guerre sur mer ont profondément évolué. Marginalisée, la marine de l'État français est passée à côté des innovations technologiques qui ont révolutionné les combats navals (asdic, radar...). De même, les équipages manquent d'expérience opérationnelle dans les domaines de lutte qui ont été renouvelés au cours des hostilités (opérations aéronavales et amphibies, lutte anti-sous-marine et anti-aérienne).

En outre, contrairement à ce qu'écrit dans les années 1960 dans un ouvrage devenu une référence Henri Noguères, ancien Résistant devenu journaliste et historien, Toulon n'est pas «la plus spectaculaire “opération suicide" de l'histoire de la marine de tous les temps et de tous les pays ${ }^{4}$. Pour impressionnantes qu'aient été les pertes de novembre 1942, elles s'avèrent pratiquement moitié moindre de celles de la marine impériale allemande à Scapa Flow, le 21 juin $1919^{5}$. Avec quatre cent mille tonnes envoyées par le fond de cette baie des îles Orcades, au nord de l'Écosse, c'est bien le sabordage de la Hochseeflotte qui reste à ce jour le plus important de l'Histoire. D'autant que cette flotte est exclusivement constituée de bâtiments de combat opérationnels, ce qui est loin d'être le cas de l'ensemble toulonnais, on l'a vu.

Le suicide de la Hochseeflotte constitue d'ailleurs une référence naturelle pour les contemporains, à l'exemple du vice-amiral Le Luc, un proche de Darlan, chef d'état-major des forces navales de Vichy entre novembre 1942 et mars $1943^{6}$. Comme l'ensemble des officiers, Le Luc se réfère à un code de l'honneur commun à toutes les marines, qui impose au commandant d'un bâtiment de le détruire plutôt que de le laisser tomber entre des mains étrangères. La Marine nationale

\footnotetext{
4. H. Noguères, Le Suicide de la flotte française à Toulon, Paris, Robert Laffont, 1961, p. 9. Une vision qui est encore largement celle du seul documentaire télévisé consacré au sabordage, pourtant réalisé il y a quelques années seulement : "D'une guerre à l'autre. Toulon 1942, le sabordage de la marine française ", réalisateurs Adila BennedjäZou et Christophe Talczewski, produit par Point du jour et France télévisions avec le soutien du ministère de la Défense, première diffusion France 3, jeudi 26 novembre 2015.

5. Et ce, même si, contrairement aux Allemands à Toulon, les Britanniques réussissent à interrompre l'opération : seuls cinquante-deux des soixante-quatorze bâtiments internés sont coulés, soit environ quatre cent mille tonnes sur cinq cent quarante mille. F.-E. Brézet, "Juin 1919, la flotte de haute mer allemande se saborde à Scapa Flow ", Revue historique des armées $n^{\circ}$ 3, 1999, pp. 53-61.

6. SHD-MV, 3 BB8 CE 11, mémoires de l'amiral Le Luc à propos du sabordage de Toulon, rédigées en 1943-1944.
} 
avait d'ailleurs déjà procédé à des actions de ce type dès juin 1940 . Devant l'avancée des forces allemandes, toutes les unités incapables de s'échapper des bases du littoral atlantique avaient été détruites, en particulier celles en réparation ou en construction, soit un total d'environ cent soixante-dix mille tonnes ${ }^{7}$.

Par la suite, les marins français ont eu à Toulon la même réaction que leurs camarades danois quelques mois plus tard : confrontés à une tentative allemande similaire (opération Safari), ils sabordent la majorité de leurs navires dans le port de Copenhague, le 29 août $1943^{8}$. Une dizaine de jours plus tard, quand l'Italie rejoint le camp allié à la suite de la signature de l'armistice de Cassibile (3 septembre), la Regia Marina fait partiellement de même. Si l'essentiel de ses forces réussit à quitter la grande base de La Spezia, le 9 septembre, pour rallier Malte, les bâtiments qui ne peuvent prendre la mer sont sabordés. Jusqu'au 18 septembre, un peu partout en Italie et dans le monde, ce sont environ cent mille tonnes qui sont ainsi détruites, dont une moitié de bâtiments de combat ${ }^{9}$. Le drame de Toulon s'inscrit ainsi dans une pratique récurrente au cours du second conflit mondial, même si ce sabordage tranche par son ampleur.

La première destruction volontaire d'un bâtiment de combat est d'ailleurs le fait de la Kriegsmarine. Le 17 décembre 1939, le cuirassé de poche Admiral GrafSpee est coulé par son équipage après être sorti du port de Montevideo, en Uruguay, où il s'était réfugié après la bataille du Rio de la Plata. À l'autre extrémité de la période, les Allemands, alors sur le point d'abandonner Toulon, fin août 1944, font de même avec leurs deux derniers $U$-Boote ${ }^{10}$. Cette décision annonce le dénouement de la fin de la guerre. Que ce soit dans le cadre de l'opération Regenbogen, lancée le 30 avril 1945 par l'amiral Dönitz pour éviter aux sous-marins de la Kriegsmarine de tomber entre les mains alliées, ou du fait d'initiatives locales, plus de deux cents $U$-Boote seront sabordés, représentant un total supérieur à deux cent mille tonnes ${ }^{11}$. Des pertes voisines de celles enregistrées à Toulon...

7. Ph. Lasterle, "Autopsie d'un exode maritime : l'évacuation des ports par la marine », in Ch. Lévisse-Touzé (dir.), La Campagne de 1940, Paris, Tallandier, 2001, pp. 261-288.

8. Trente-deux bâtiments sont sabordés sur un total d'une cinquantaine, totalisant quinze mille tonnes environ; six unités réussissent à s'échapper, quatorze sont saisies intactes par les Allemands. H. P. Willmott, Last Century of Sea Power. Vol. 2, From Washington to Tokyo, 1922-1945, Bloomington and Indianapolis, Indiana University Press, 2010, pp. 297-299.

9. À la différence du cas français, où les bâtiments de combat constituent l'immense majorité des pertes. De fait, on compte peu de grandes unités parmi les navires italiens sabordés, contrairement à ce qui se passe à Toulon : V. O'Hara et E. Cernuschi, Dark Navy. The Italian Regia Marina and the Armistice of 8 September 1943, Ann Harbour, Nimble Books, 2009

10. M. Saibène, Toulon et la Marine, du sabordage à la Libération, Nantes, Marines éditions, p. 112.

11. Ch. Madsen, The Royal Navy and German naval disarmament, 1942-1947, London, Frank Cass, 1998, p. 51. Ce tonnage doit être rapporté à celui des U-Boots saisis par les Britanniques et qu'ils sabordent finalement au large de Lisahally, en Irlande du Nord, ou du Loch Ryan, sur la côte ouest de l'Écosse, entre le 17 novembre 1945 et le 11 février 1946. A l'issue de l'opération Deadlight, cent vingt et un U-Boots ont été coulés, représentant un total de près de quatre-vingtdix mille tonnes. 
Par ailleurs, le geste des marins français doit être rapporté à l'attitude de leurs camarades des autres armées restées fidèles à Vichy. D'autant que le sabordage intervient à la suite de l'invasion de la zone libre et coïncide avec la dissolution de l'armée d'armistice ordonnée par Hitler. La différence de réaction est flagrante. À de rares exceptions près, les armées de terre et de l'air n'opposent aucune résistance et abandonnent intact leur matériel. Mille deux cent soixante et onze avions de combat sont saisis par les forces allemandes : après réforme, plus de sept cents appareils seront effectivement réemployés par la Luftwaffe (cinq cent quarante) et la Regia Aeronautica (cent soixantesept) ${ }^{12}$. Par ailleurs, en additionnant les matériels autorisés par les conventions d'armistice et ceux camouflés mais finalement tous découverts par l'occupant, on peut estimer que huit cents à neuf cents canons sont saisis, six mille mortiers, neuf mille à douze mille mitrailleuses, et entre cent cinquante mille et deux cent mille armes individuelles ${ }^{13}$.

\section{Premières réactions : un sacrifice jugé utile}

L'annonce du sabordage provoque immédiatement un déluge de réactions. À l'image de ce qu'écrit L'Humanité, le «sacrifice héroïque ${ }^{14}$ des marins de Toulon est partout mis en exergue côté allié. Sans le moindre doute, le sabordage est interprété comme une défaite pour l'Axe. Le service de presse de la France combattante y voit ainsi «un acte efficace pour notre victoire ${ }^{15}$. Au printemps 1943, le célèbre magazine américain Life considèrera même qu'il s'agit de la contribution la plus importante de la France à la cause alliée depuis le début du conflit ${ }^{16}$.

Cette contribution a néanmoins un goût amer. Chez de Gaulle domine une forme de soulagement devant une action dramatique, mais qui a «épargné à la patrie la honte suprême de voir ses vaisseaux devenir des vaisseaux ennemis ${ }^{17}$. «Nous avons évité le pire », résume le journaliste Pierre Limagne dans son journal personnel au

12. Cl. d'Abzac-Épezy, L'Armée de l'air des années noires. Vichy 1940-1944, Paris, Economica, 1998, p. 264.

13. Synthèse établie en particulier d'après R. Paxton, L'Armée de Vichy. Le corps des officiers français, 1940-1944, Paris, Tallandier, 2004, p. 63 et pp. 307-315. II faut y ajouter une soixantaine d'automitrailleuses équipées d'un canon de $37 \mathrm{~mm}$, cent vingt voitures de liaison et environ cent cinquante motocyclettes. Les canons de campagne sont d'un calibre inférieur ou égal à $75 \mathrm{~mm}$.

14. L'Humanité. Édition clandestine, zone Nord, 4 décembre 1942.

15. SHD-MV, 273 TTD, Bulletin d'informations générales $n^{\circ} 40,28$ janvier 1943

16. Life, 15 mars 1943.

17. Discours prononcé à la radio de Londres, 27 novembre 1942 (Ch. de Gaulle, Discours et messages. T. I, Pendant la guerre. Juin 1940-janvier 1946, Paris, Plon, 1970, p. 244). 
lendemain du drame, avant de souligner l'ampleur des destructions matérielles ${ }^{18}$. La perte de tous les bâtiments des FHM est en effet lourde de conséquences pour l'avenir de la marine, d'autant que la guerre est loin d'être terminée. Dans son Journal politique, le comte Ciano, ministre italien des Affaires étrangères, ne s'y trompe pas : "C'est une puissance navale qui dispar[aît] de la Méditerranée pour de longues années $\gg$ note-t-il le 27 novembre ${ }^{19}$.

Dans les jours qui suivent, la stupeur laisse la place à l'émotion. Si le sabordage est une victoire, c'est une victoire endeuillée. Le bulletin des FNFL ne manque d'ailleurs pas de remarquer que l'événement s'est déroulé en novembre, «mois de la fête des morts $»^{20}$. Le 2 décembre, la marine gaulliste organise un service religieux en la chapelle militaire St. Peter de Londres. Plusieurs unités engagées dans la bataille de l'Atlantique ont devancé cette initiative, mettant leur pavillon en berne et célébrant en mer des cérémonies funéraires ${ }^{21}$. Pour beaucoup, côté Alliés, la rade de Toulon s'est transformée en un cimetière «voilé du crêpe des fumées d'incendies $\gg^{22}$.

Les commentaires relatifs au sabordage partagent un dernier point commun : l'idée que ce geste tranche le nœud gordien qui retenait prisonnières la France et sa marine depuis plus de deux ans. Aux yeux des adversaires de Vichy, le sabordage a pour vertu principale de mettre un terme définitif à la fiction d'indépendance revendiquée par l'État français et de réunir à nouveau l'ensemble de la nation dans la lutte. Ainsi que le résume Pierre Villon, secrétaire général du Front national, organisation proche du parti communiste, dans Les Lettres françaises, «une nation capable de tels sacrifices n'est pas vaincue, n'est pas déchue $\gg^{23}$. Toulon devient même le symbole de l'unité retrouvée des Français, comme le suggère Radio-Moscou, dès le 28 novembre $1942^{24}$.

Sans surprise, l'interprétation est très différente à Vichy. L'événement prive l'État français de sa dernière carte majeure, d'autant qu'il est concomitant de la dissolution de l'armée d'armistice. Il est donc très difficile de lui donner un sens. Revendiquer une victoire serait de nature à offrir un argument de plus aux Allemands pour justifier leur tentative de saisir la flotte. Inversement, y voir un échec reviendrait à

18. P. Limagne, Éphémérides de quatre années tragiques, t.2, Paris, Bonne Presse, 1947, p. 915.

19. G. Ciano (comte), Journal (1939-1943), Paris, La Baconnière-Payot, 2013, p. 690 (mention du 27 novembre 1942).

20. Bulletin des Forces navales françaises libres $n^{\circ} 12$, janvier 1943.

21. F. Flohic (amiral), Ni chagrin ni pitié. Souvenirs d'un marin de la France libre, Paris, Plon, 1985, p. 160.

22. L'Écho d'Alger, 28 novembre 1942.

23. "La leçon de Toulon", Les Lettres françaises $n^{\circ} 4$, décembre 1942. Document publié par F. Eychart et G. Aillaud, Les Lettres françaises et les étoiles dans la clandestinité, 1942-1944, Paris, Le Cherche Midi, 2008.

24. "Toulon", 28 novembre 1942 (J.-R. Bloch, De la France trahie à la France en armes. Commentaires à Radio Moscou, Paris, Éditions sociales, p. 211). 
condamner les chefs de la marine qui, dans leur écrasante majorité, ont toujours apporté un soutien sans faille au régime. Ainsi, le Journal de la marine marchande et de l'Empire français se borne à en relater les éléments factuels déjà connus, tandis que Mer et Colonies, la revue de la Ligue maritime et coloniale française, ne fait, elle, pratiquement aucune référence à l'événement ${ }^{25}$.

Dans un premier temps, la censure de Vichy oblige la presse à reproduire la lettre qu'Hitler a envoyée à Pétain pour justifier la tentative de mainmise sur Toulon. Le Führer y rejette la faute sur les officiers de marine accusés de forfaiture ${ }^{26}$. Ce réquisitoire pousse le commandant des FHM, l'amiral de Laborde, à diffuser une mise au point. Il dément avoir donné l'ordre de ne pas combattre les Anglo-Américains et revendique n'avoir jamais pris la moindre disposition d'appareillage pour fuir la rade ${ }^{27}$. Ce document, qui sera ensuite largement utilisé dans l'instruction de son procès, va nourrir les critiques à l'égard du sabordage.

Mais, dans l'immédiat, les premiers à considérer que Toulon est un échec sont les éditorialistes de la presse collaborationniste parisienne. Dans Les Nouveaux Temps, Jean Luchaire se déchaîne contre la «désobéissance» des officiers et contre la «trahison installée partout ${ }^{28}$. Quant à Marcel Déat, le chef du Rassemblement national populaire, il invite les lecteurs de L'Eeuvre à «pleur[er] 》 devant l'«indignité » et la «trahison» des chefs de la marine ${ }^{29}$. Le sabordage marque le naufrage de la politique que préconisaient les journaux collaborationnistes. Pour ces derniers, la marine s'est sabordée à cause des «excitations perfides qui la poussaient à la résistance active ou à la dissidence $\gg^{30}$.

Au-delà du caractère caricatural des analyses du Matin, un quotidien rallié de longue date à la collaboration, l'emploi de ces termes interroge. Peut-on qualifier la destruction de la flotte d' «acte de résistance $\gg^{31}$ à la manière dont Le Figaro le fera en juillet 1946 ? Ce n'est officiellement pas le cas pour la marine à cette date. Au contraire, l'institution valorise et récompense les rares unités qui ont refusé le sabordage. Dès le 20 décembre 1942, le sous-marin Marsouin est par exemple cité à l'ordre de l'armée de mer pour avoir réussi à quitter

\footnotetext{
25. Respectivement Journal de la marine marchande et de l'Empire français, jeudi 3 décembre 1942, pp. 1464-1466 et Mer et Colonies. Revue de la Ligue maritime et coloniale française $n^{\circ}$ 322, $1^{\text {er }}$ trimestre 1943.

26. Consigne ${ }^{\circ} 1004$, citée par P. Limagne, op. cit., p. 914.

27. SHD-MV, 273 TTD, ordre du jour de l'amiral de Laborde, 28 novembre 1942.

28. Les Nouveaux Temps, 29 et 30 novembre 1942.

29. L'Euvre, 28 et 29 novembre 1942.

30. Le Matin, 28 novembre 1942.

31. Le Figaro, 30 juillet 1946.
} 
Toulon $^{32}$. Sans surprise, les combattants de l'ombre vont beaucoup plus loin. Ainsi, dans son Journal des années noires, l'écrivain Jean Guéhenno fustige presque immédiatement l'attitude de la marine qui «eût mieux fait de ne pas cesser le combat depuis deux ans : on n'a pas de mérite à être dupe ${ }^{33}$. Ce jugement cinglant annonce une nouvelle lecture du sabordage.

\section{De «l'héroïque sabordage» au « suicide sans honneur » ${ }^{34}$}

Dès 1943, dans un reportage qui est aussi la première histoire du déroulement des opérations, le journaliste Yves Farge, l'un des fondateurs du mouvement de résistance Franc-Tireur, avance l'idée que le sabordage de la flotte est la conséquence de la trahison du haut-commandement ${ }^{35}$. Cette question des responsabilités cristallise rapidement le débat. À l'occasion du premier anniversaire du drame de Toulon, le nouveau commissaire à la Marine du Comité français de libération nationale (CFLN), Louis Jacquinot, issu de la droite modérée, ne fait pas mystère de ses intentions. Évoquant la perte des bâtiments, il annonce que «les quelques chefs aveugles ou indignes, coupables de ne les avoir pas sauvés en donnant en temps voulu les ordres nécessaires, seront jugés ${ }^{36}$. Entre-temps, il est vrai, le ralliement à la cause alliée de l'essentiel des forces de la Regia Marina, en septembre 1943, a témoigné de manière spectaculaire qu'une alternative était possible.

Le tournant dans l'interprétation du 27 novembre se situe durant l'immédiat après-guerre : l'épuration est son catalyseur. L'éditorial intitulé «La France humiliée » publié le 12 août 1945 dans Combat par Georges Bernanos donne le ton. Au lendemain des réquisitions du procès Pétain, l'écrivain ne mâche pas ses mots : «En tant que démonstration civique, [ce procès] vaut exactement ce qu'a valu, en tant que démonstration d'héroïsme militaire, le grotesque et désastreux sabordage de Toulon ${ }^{37}$. » Au début de l'été, Maurice Thorez célébrait encore lors du $\mathrm{X}^{\mathrm{e}}$ congrès du $\mathrm{PGF}$ «le sacrifice héroïque de

32. SHD-MV, 198 GG² 1, citation à l'ordre de l'armée de mer n 134, signée par l'amiral Darlan, 20 décembre 1942.

33. J. Guéhenno, Journal des années noires 1940-1944, Paris, Gallimard, "Folio", 2014 (1947 pour la première édition), p. 324. Mention du $1^{\text {er }}$ décembre 1942.

34. A. Piernic, L'Héroïque Sabordage de la flotte française. Toulon 27 novembre 1942, Paris, Éditions de l'lle-de-France, 1945; L. Moënard, Un suicide sans honneur. Toulon 1942, Rennes, Éditions Ouest-France, 2013.

35. Y. Farge, Toulon, Paris, Éditions de Minuit, 1943.

36. Ordre du jour de Louis Jacquinot à l'occasion de l'anniversaire du sabordage de la flotte de Toulon, publié dans Bulletin de la Marine française $n^{\circ} 24$, janvier 1944.

37. Combat, 12 août 1945. Ces propos sévères font suite à ceux tenus dès le mois de décembre 1942 (G. Bernanos, "Le scénario de Toulon ", Essais et Écrits de combat, t. 2, Paris, Gallimard, "Bibliothèque de la Pléiade», 1995, pp. 476-478). 
nos marins à Toulon ${ }^{38}$. Après la condamnation du Maréchal, tombée le 15 août 1945, le ton change. À l'occasion du troisième anniversaire du sabordage, L'Humanité oppose désormais les équipages et les cadres de la marine. Aux premiers, le quotidien réserve ses louanges pour avoir coulé les navires, tandis que les seconds sont soupçonnés d'avoir été tentés de livrer la flotte à Hitler ${ }^{39}$.

Une étape supplémentaire est franchie avec le «procès des amiraux», qui se déroule à l'été 1946 puis au printemps 1947. Parmi les anciens responsables de la marine jugés à cette occasion, l'amiral Jean de Laborde, commandant les FHM, et le vice-amiral André Marquis, préfet maritime de Toulon, sont lourdement sanctionnés. Si le sabordage a été au cœur des débats, il n'est en revanche pas central dans les attendus du jugement. Il est pourtant ce qui est retenu par la presse, car l'audience a démontré la possibilité technique pour la flotte d'appareiller dans les jours qui ont précédé le 27 novembre. Mais personne n'en a donné l'ordre, laissant les amiraux s'enfermer dans une impasse qu'ils n'ont pas voulu surmonter...

De plus, la révélation des projets nourris par Laborde de lever une légion de volontaires pour reprendre le Tchad, rallié à la France combattante, offre un nouvel élan à la thèse de la trahison, tout en la faisant basculer de l'extrême droite à l'extrême gauche. Même si cet élément n'a rien à voir avec le sabordage, le chef des FHM est dorénavant pour L'Humanité l' «amiral félon» ou le «sabordeur » et le geste est frappé d'opprobre ${ }^{40}$. En ce domaine, le temps n'adoucira pas les jugements.

Dans les décennies qui suivent, le sabordage va cesser en effet d'être un échec pour se transformer en faute : Toulon devient la métaphore de la faillite morale de Vichy. «La France dans sa bêtise, sa vertueuse et capitale bêtise, c'était Toulon dans la quiétude de ses amiraux endormis. » Edmonde Charles-Roux, ancienne Résistante et récent prix Goncourt, donne le ton avec les pages cinglantes qu'elle consacre à l'événement, en 1971, dans son roman Elle Adrienne ${ }^{41}$. Cette interprétation se diffuse dans le grand public, car elle est nourrie du talent de vulgarisateurs comme Alain Decaux qui, quarante ans après les faits, juge «légitime que l'Histoire ait réservé à l'amiral de Laborde toute sa sévérité ${ }^{42}$. L'image de la marine en est durablement affectée chez les élites comme dans l'opinion.

38. Rapport au Xe congrès national du PCF, $26-30$ juin 1945, cité dans M. Thorez, Une politique de grandeur française, Paris, Éditions sociales, 1945, p. 300.

39. L'Humanité, 28 novembre 1945.

40. Ibid.

41. E. Charles-Roux, Elle Adrienne, Paris, Grasset, 1971, p. 309.

42. A. Decaux, L'Histoire en question, Paris, Perrin, 1982, p. 109 


\section{La défense d'un "pavillon sans tâche "}

Avant même la fin des hostilités, le ministre de la Marine, Louis Jacquinot, s'est pourtant employé à inverser la tendance. Le retour solennel de la flotte à Toulon, le 13 septembre 1944, qu'il préside en personne, illustre parfaitement cette ambition. Afin d'exorciser le sabordage, toutes les unités disponibles pénètrent dans la rade où gisent encore des dizaines d'épaves. Les bâtiments arborent au sommet de leur mât la traditionnelle flamme de guerre, longue d'un mètre supplémentaire pour chaque mois passé en campagne. Celle du navire amiral, le croiseur Georges Leygues, parti de Toulon le 9 septembre 1940, fait près de cinquante mètres de long... Spectaculairement mise en scène, la fiction d'un engagement continu au combat est ainsi officiellement établie. Embarqué ce jour-là, Roger Chapelet, l'un des grands peintres de marine du $\mathrm{XX}^{\mathrm{e}}$ siècle, en donnera une représentation magnifiée ${ }^{43}$. La perspective retenue, celle des Toulonnais observant l'arrivée de la flotte, prive le tableau de toute référence au cimetière naval que découvrent les équipages des bâtiments entrant dans la rade et qui constitue comme le négatif de l'escadre victorieuse. Un mois après le débarquement de Provence, dans la réussite duquel la marine a joué un rôle important, la page est tournée au prix d'une mise en scène habile et de beaucoup d'ambiguïtés.

Cette stratégie destinée à faire oublier l'événement se manifeste aussi à l'occasion de l'exposition accueillie à Paris, au musée de la Marine, quelques semaines plus tard. Intitulée «La marine au combat», elle vise à valoriser la participation de l'institution à l'effort de guerre. Pour cela, l'action des FNFL et des marins dans la Résistance est mise en avant, tout en passant sous silence les éléments gênants. Si le sabordage est présent dans la brochure qui accompagne cette exposition, il l'est uniquement à l'aune des réactions étrangères au lendemain du drame. Indirectement, les encarts consacrés aux sous-marins Casabianca et Marsouin l'évoquent aussi, mais pour souligner l'héroïsme de leur évasion de Toulon ${ }^{44}$.

Un pas supplémentaire est franchi l'année suivante quand le gouvernement Bidault décerne la médaille de la Résistance à ces deux submersibles, ainsi qu'aux sous-marins Glorieux et Vénus qui se sont également échappés de la nasse toulonnaise ${ }^{45}$. Ces bâtiments appartiennent aux rares unités ne relevant pas des Forces françaises

\footnotetext{
43. R. Chapelet, Retour de la flotte française à Toulon en 1944, gouache sur papier marouflé, 139 × $300 \mathrm{~cm}$, Toulon, musée national de la Marine.

44. La Marine au combat, Paris, Marine nationale, 1945, document non paginé.

45. Le seul sous-marin évadé de Toulon à ne pas avoir obtenu cette récompense est l'Iris, qui a rejoint Barcelone où il est resté interné.
} 
libres à être distinguées par cette décoration. Désormais, le geste hérö̈que n'est donc plus le sabordage mais la sortie de la rade, considérée comme un acte de résistance à célébrer et à commémorer. L'évolution de la muséographie du musée national de la Marine le confirme. Dans une collection permanente où dominent largement, à partir du début des années 1950, les souvenirs de la marine de Vichy, le Casabianca est mis en avant, non pour son rôle dans la libération de la Corse, mais pour son évasion de Toulon ${ }^{46}$.

Avec son rythme propre, l'historiographie de la marine pendant la Seconde Guerre mondiale participe initialement de cette stratégie d'évitement et de reconstruction mémorielle ${ }^{47}$. Elle privilégie en effet une approche opérationnelle, qui permet de livrer une vision en apparence dépassionnée et apolitique du conflit. Ce phénomène est facilité par une écriture de l'histoire longtemps préemptée par d'anciens acteurs des événements très engagés en faveur de la Révolution nationale. Jusque dans les années 1970, le contre-amiral (2S) Paul Auphan, secrétaire d'État à la Marine jusqu'à la veille du sabordage, est ainsi au centre d'une nébuleuse qui bénéficie de relais au sein de l'institution. C'est en particulier le cas au Service historique de la marine (SHM) et au musée de Chaillot, grâce aux fonctions qu'y occupe successivement le médecin de marine Hervé Cras, son ancien secrétaire particulier à Vichy en $1942^{48}$. Sous le pseudonyme de Pierre Mardyck, ce dernier est un collaborateur régulier de L'Indépendance française, le «bulletin bi-mensuel du nationalisme français» que dirige Jean Madiran. Son ambition affichée est d'exonérer l'institution et son ex-patron de leurs responsabilités. "Les chefs de la marine n'ont pas failli » résume-t-il ${ }^{49}$. Usant d'une rhétorique chère aux nostalgiques de l'État français, il prétend dès la Libération que la décision de saborder les bâtiments aurait évité à la France la mainmise nazie. Ils développera en 1956 cette thèse dans le plaidoyer pro domo qu'il publiera sous son nom de plume ${ }^{50}$.

Ce courant apologétique, distinguant un «bon» et un «mauvais» Vichy, est également illustré par Le Sabordage de la flotte (1954) de Pierre Varillon, l'ex-chroniqueur maritime de L'Action française. Ou encore avec Les Vingt Derniers Jours de la flotte, écrit par un autre ancien du cabinet

46. T. Vaisset, "L'impuissance navale au musée? La "Royale" et la Seconde Guerre mondiale au musée national de la Marine", Neptune au musée. Puissance, identité et conflits dans les musées maritimes et navals, in P. Louvier (sd). Presses universitaires de Rennes, 2014, pp. 97-115.

47. J.-B. Bruneau, "Gloria victis. L'écriture de l'histoire navale de la Seconde Guerre mondiale ", Revue d'histoire maritime $n^{\circ} 10 / 11,2010$, pp. 357-366.

48. Affecté à la section historique du SHM à partir de 1951, il devient chef du service études du musée à sa retraite en 1969.

49. L'Indépendance française. Bulletin bimensuel du nationalisme français $n^{\circ} 13$, avril 1947.

50. J. Mordal, La Marine à l'épreuve. De l'armistice de 1940 au procès Auphan, Paris, Plon, 1956. 
Auphan à Vichy, le capitaine de frégate Vulliez. Vingt ans après le drame, il n'hésitera pas à affirmer au sujet de Laborde : «Quelles qu'aient pu être ses erreurs passées, la grandeur du dénouement a tout effacé ${ }^{51}$.»

Noyant les rares productions un tant soit peu critiques, comme celle de l'ambassadeur et ancien Résistant Albert Kammerer ${ }^{52}$, ces ouvrages mettent en particulier un point d'honneur à souligner que le sabordage est la concrétisation de la promesse faite par Darlan à Churchill, en juin 1940, de ne jamais livrer la flotte à une puissance étrangère. Cette veine ne s'épuise pas, puisqu'elle est encore exploitée en 1986 par le contre-amiral (2S) Wassilieff dans un livre publié à l'heure de la retraite et au titre en forme de manifeste : Un pavillon sans tâche $e^{53}$.

La perspective du cinquantième anniversaire de l'événement tend néanmoins à favoriser une approche plus balancée. C'est le cas du livre rédigé par le journaliste Jean-Jacques Antier, richement documenté, ou de l'ouvrage écrit à quatre mains par le vice-amiral (2S) François Flohic, ancien FNFL, et le commissaire de la Marine (H) Jacques Raphäel-Leygues, ancien «Barbaresque» devenu député puis ambassadeur ${ }^{54}$. Le portrait croisé qu'ils dressent des amiraux Darlan et de Laborde se veut œuvre d'apaisement, tournée vers l'avenir : «Nous pensons fortement, si l'on veut que la marine se retrouve vraiment dans une marine d'avenir, qu'il faut une fois pour toutes fermer la page du sabordage ${ }^{55}$. $\gg$ Ces propos ne peuvent que rencontrer l'adhésion de l'institution. À l'image de ceux du contreamiral (2S) Remi Monaque qui, vingt-cinq ans plus tard, livre une analyse équilibrée de la «tragédie du sabordage» qui n'esquive pas les difficultés ${ }^{56}$.

\section{La recomposition de la mémoire institutionnelle}

La Marine nationale est restée en retrait, d'autant qu'elle trouvait son compte dans cette approche événementielle et en apparence apolitique, également portée par son service historique. Or, Toulon ne peut être compris sans référence à l'adhésion de la grande majorité

51. A. Vulliez, Les Vingt Derniers Jours de la flotte, Paris, Presses de la Cité, 1963, p. 286.

52. A. Kammerer, La Passion de la flotte. De Mers el-Kébir à Toulon, Paris, Fayard, 1951.

53. A. Wassilieff, Un pavillon sans tâche. De l'armistice au sabordage, la vérité, Paris, Grasset, 1986.

54. J.-J. Antier, La Flotte se saborde, Toulon 1942, Paris, Presses de la Cité, 1986; J. Raphël-Leygues et F. Flohic, Darlan Laborde. L'inimité de deux amiraux, Brest, Éditions de la Cité, 1990. Dans un registre relativement proche, voir également le livre de G. Perrier, Le Suicide de la flotte française. Toulon, 27 novembre 1942, Paris, Pygmalion, 2010.

55. J. Raphël-Leygues et F. Flohic, op. cit., p. 15.

56. R. Monaque, Une histoire de la marine de guerre française, Paris, Perrin, 2016, pp. 428-437. 
des chefs de l'époque aux idéaux de la Révolution nationale ${ }^{57} \ldots$ Néanmoins, trois éléments distincts montrent qu'une rupture s'opère dans les années 2000. Par leur caractère contradictoire, ils témoignent aussi de l'éclatement de la mémoire du sabordage au sein de l'institution.

En 2003, sur proposition du commandant de la Force océanique stratégique, le chef d'état-major de la marine, l'amiral Jean-Louis Battet, fait de la date du 27 novembre la journée du sous-marinier ${ }^{58}$. L'objectif est double. Il s'agit tout d'abord de donner une image attractive de l'arme sous-marine, qui est constamment à la recherche d'un personnel suffisant, tant sur le plan quantitatif que qualitatif. Mais c'est aussi l'occasion de mettre en valeur cette spécialité, alors que se dessine la perspective du renouvellement des sous-marins nucléaire d'attaque (SNA).

Néanmoins, la date retenue interpelle. Dans la droite ligne de la position qui avait prévalu à Alger dès le lendemain du drame, puis à la Libération, l'accent est de nouveau mis sur l'évasion héroïque d'une partie des sous-marins basés à Toulon. Ces cinq unités sont cette fois encore l'arbre résistancialiste qui cache la forêt des bâtiments sabordés. Plus surprenant, le commandant de l'équipage bleu du SNA Casabianca, à l'origine lointaine de la journée du sous-marinier, reprend dans une tribune publiée par la suite les arguments pro domo du contre-amiral Auphan ${ }^{59}$.

Á la fin des années 2000, la rénovation de l'espace du musée de la Marine de Toulon consacré à la ville pendant le conflit est initialement l'objet d'un désaccord entre le directeur de l'institution, le vice-amiral (2S) Jean-Noël Gard, et la conservatrice de l'antenne locale, Cristina Baron. Le premier, officier général à la retraite, souhaite valoriser en priorité la libération du port, tandis que la seconde entend donner toute sa place au sabordage, option qui prévaut finalement ${ }^{60}$. Face au tableau monumental de Chapelet célébrant le retour de la flotte, une vitrine présente une collection de photographies prises au lendemain du sabordage. Celles-ci sont accompagnées par toutes les publications relatives au sujet, dont les titres souvent contradictoires permettent au visiteur de mesurer la complexité de la mémoire de l'événement.

57. Voir en particulier l'ouvrage tiré de sa thèse par 0. Girardin-Thibeaud, Les Amiraux de Vichy, Paris, Ministère de la Défense/Nouveau Monde Éditions, 2016.

58. J. de Préneuf, «Un héros pour tous. Jean Lherminier et le Casabianca, figures emblématiques du mythe résistancialiste de 1942 à nos jours", in Cl. d'Abzac-Épezy et J. de Préneuf (sd), Héros militaire, culture et société (XIXe-XXe siècle), Villeneuve d'Ascq, 2012, publié en ligne : http://hleno.revues.org/249. Ä cette date, le commandant de la Force océanique stratégique est le vice-amiral d'escadre Thierry d'Arbonneau.

59. A. d'Ales de Corbet (capitaine de frégate), "Le commandant Lherminier, figure emblématique des forces sous-marines et exemple de commandement ", Bulletin du CESM n ${ }^{\circ} 35$, novembre 2006, pp. 73-77.

60. Entretiens de Thomas Vaisset avec Cristina Baron, mai et novembre 2015. 
Surtout, l'ensemble est mis en perspective par un film documentaire d'une dizaine de minutes, qui mêle images d'archives et témoignages indirects, le tout appuyé par l'éclairage historique d'un spécialiste de la période, Jean-Marie Guillon, professeur à l'université d'Aix-Marseille. Cette nouvelle muséographie réussie annonce un changement de regard de l'institution sur le sabordage.

Le tournant est un article publié en 2009 par le vice-amiral Marin Gillier, commandant la force maritime des fusiliers marins et commandos, consacré aux valeurs de la Marine nationale ${ }^{61}$. Ancien commando lui-même, il n'hésite pas à y évoquer le «déshonneur»du sabordage. Les termes employés par cet officier, né quinze ans jour pour jour après les faits, sont forts. Tout en refusant de stigmatiser le commandement de l'époque, il «récuse cet héritage» et considère que le geste fut la conséquence de l'《acceptation de la défaite». À ses yeux, le drame doit nourrir une réflexion sur le processus de sélection des chefs militaires, où les critères académiques priment trop souvent sur la valeur humaine des officiers.

Trois ans plus tard, cette prise de parole pousse le chef d'état-major de la marine, l'amiral Bernard Rogel, sous-marinier d'origine, à accorder un bref entretien centré sur le sabordage à l'occasion de son soixante-dixième anniversaire. C'est la première fois que la plus haute autorité militaire de la marine évoque publiquement le drame de Toulon. Nuançant les propos du vice-amiral Marin Gillier, il rappelle que «le vrai déshonneur eut été de livrer intacte la flotte à l'ennemi », reprenant ainsi l'argumentaire développé par le général de Gaulle au lendemain des événements. Mais il souligne également que le sabordage est la conséquence de l' «effondrement moral de l'État français » et de son engagement «sur la voie du renoncement », à l'opposé du choix des Français libres ${ }^{62}$. Des propos sans ambiguïté, même si le reste de l'analyse demeure prudente, dans un souci manifeste de ne pas réveiller les vieilles blessures.

On l'aura compris, Toulon relève de ce «passé qui ne passe pas », en tout cas difficilement ${ }^{63}$. Les faits dans leur matérialité ont pourtant été établis jusqu'à l'excès. On n'ignore plus rien du déroulement de la fatidique journée du 27 novembre 1942 comme de celles qui y ont conduit. Mais le traitement de l'événement a été saturé d'enjeux moraux et mémoriels. Ce qui explique en bonne part pourquoi, en dépit du nombre d'ouvrages consacrés au sujet, aucun ne s'est imposé

\footnotetext{
61. M. Gillier (vice-amiral), "Les quatre valeurs de la marine dans les opérations", Le Lien. Le magazine des fusiliers marins et commandos $n^{\circ} 66,2^{e}$ semestre 2009, pp. 6-9.

62. Disponible en ligne : http://www.marianne.net/blogsecretdefense/Amiral-Rogel-le-vrai-deshonneur-eut-ete-de-livrerintacte-la-flotte-a-I-ennemi_a850.html

63. Pour reprendre la célèbre formule d'H. Rousso et É. Conan, Vichy, un passé qui ne passe pas, Paris, Fayard, 1994.
} 
véritablement comme la référence indiscutable. Focalisés sur le drame, la plupart des auteurs ont eu du mal à garder la distance nécessaire.

Toulon est, pour une part, un sabordage parmi d'autres. À l'aune des deux conflits mondiaux, du second en particulier, l'épisode est loin d'être unique. La destruction volontaire d'un navire ou d'une flotte est un acte exceptionnel, mais classique de la guerre sur mer, qui n'a rien d'infamant en soi. En privant l'adversaire des bâtiments qu'il convoitait, le sabordage limite spectaculairement son succès et préserve l'honneur de ceux qui l'opèrent. De ce point de vue, Toulon n'est pas un échec, au contraire. La capitulation de Bizerte, une dizaine de jours plus tard, en fournit un parfait contre-exemple ${ }^{64}$.

À l'opposé de ce qui était redouté par les Alliés, en conformité avec ce qui avait été promis depuis juin 1940, les marins de Toulon détruisent systématiquement leurs matériels, à l'inverse des terriens et des aviateurs une quinzaine de jours plus tôt. Il est vrai que le capital rassemblé dans le port varois était d'une autre importance. Symboliquement et pratiquement, l'enjeu était bien supérieur, ce qui n'a pas peu contribué à radicaliser la situation. Mais l'attachement que les marins avaient pour leurs bâtiments, leurs commandants en tête, était également d'une autre nature : saborder son navire est une forme de violence extrême qu'un équipage s'inflige à lui-même. Pour reprendre, en l'adaptant, la fameuse distinction d'Ernst Kantorowicz, les marins tuent leur corps collectif symbolique, même si leur corps physique individuel survit ${ }^{65}$.

Cependant, à la différence de ce qui prévalait à Scapa Flow en juin 1919, ou en Baltique fin avril 1945, le conflit n'était pas fini en novembre 1942. Et les bâtiments sabordés ne le furent pas au moment de partir continuer le combat ailleurs, comme à la mi-juin 1940 pour la Marine nationale ou début septembre 1943 avec la Regia Marina. D'évidence, le sabordage est également un échec magistral. Voilà pourquoi de Gaulle en parlera, dans ses Mémoires de guerre, comme du «suicide le plus lamentable et le plus stérile qu'on puisse imaginer ${ }^{66}$. Voilà pourquoi, inaugurant la nouvelle École navale le 15 février 1965, le chef de l'État rappellera solennellement que la marine «est faite pour la guerre, [...] autrement dit pour combattre, pour s'y préparer d'abord et le cas échéant pour l'accomplir ${ }^{67}$, évocation à peine voilée de ce qui avait fait si cruellement défaut en novembre 1942.

64. Après la capitulation de l'amiral Derrien, la veille, les troupes allemandes occupent la base sans combat et saisissent intacts trois torpilleurs, trois avisos et neuf sous-marins, d'une faible valeur militaire.

65. E. Kantorowicz, Les Deux Corps du roi, Paris, Gallimard, 1989.

66. Ch. de Gaulle, Mémoires de guerre. L'Unité : 1942-1944, Paris, Plon, 1956, p. 49.

67. Ch. de Gaulle, "Allocution prononcée à l'École navale», Défense nationale et sécurité collective, hors-série "Porteavions Charles de Gaulle», 2008, p. 5 . 
Le sabordage apparaît ainsi comme un «événement Janus » ${ }^{68}$, à la fois rien qu'un échec, mais plus qu'un échec. Les deux sont indissociables et la difficulté à le reconnaître explique certainement une bonne part des limites des travaux qui ont été consacrés au sujet. On doit accepter de laisser Toulon dans cette ambivalence fondatrice. Elle offre la seule voie possible pour avancer vers une meilleure compréhension de ce qui demeure un épisode douloureux de la mémoire nationale, et pas seulement de celle de la marine. لـ 